\title{
Ultrahigh lattice thermal conductivity in topological semimetal TaN caused by large acoustic-optical gap
}

\author{
San-Dong Guo $^{1}$ and Bang-Gui Liu $^{2,3}$ \\ ${ }^{1}$ School of Physics, China University of Mining and Technology, Xuzhou 221116, Jiangsu, China \\ ${ }^{2}$ Beijing National Laboratory for Condensed Matter Physics, \\ Institute of Physics, Chinese Academy of Sciences, Beijing 100190, China and \\ ${ }^{3}$ School of Physical Sciences, University of Chinese Academy of Sciences, Beijing 100190, China
}

\begin{abstract}
Topological semimetal may have potential applications like topological qubits, spintronics and quantum computations. Efficient heat dissipation is a key factor for the reliability and stability of topological semimetal-based nano-electronics devices, which is closely related to high thermal conductivity. In this work, the elastic properties and lattice thermal conductivity of TaN are investigated by first-principles calculations and the linearized phonon Boltzmann equation within the single-mode relaxation time approximation (RTA). According to the calculated bulk modulus, shear modulus and $C_{44}$, TaN can be regarded as a potential incompressible and hard material. The room-temperature lattice thermal conductivity is predicted to be $838.62 \mathrm{Wm}^{-1} \mathrm{~K}^{-1}$ along a axis and $1080.40 \mathrm{Wm}^{-1} \mathrm{~K}^{-1}$ along $\mathrm{c}$ axis, showing very strong anisotropy. It is found that the lattice thermal conductivity of TaN is several tens of times higher than one of other topological semimetal, such as TaAs, MoP and ZrTe, which is due to very longer phonon lifetimes for TaN than other topological semimetal. The very different atomic masses of Ta and $\mathrm{N}$ atoms lead to a very large acoustic-optical band gap, and then prohibits the scattering between acoustic and optical phonon modes, which gives rise to very long phonon lifetimes. Based on mass difference factor, the WC and WN can be regarded as potential candidates with ultrahigh lattice thermal conductivity. Calculated results show that isotope scattering has little effect on lattice thermal conductivity, and that phonon with mean free path(MFP) larger than $20(80) \mu \mathrm{m}$ at $300 \mathrm{~K}$ has little contribution to the total lattice thermal conductivity. This work implies that TaN-based nano-electronics devices may be more stable and reliable due to efficient heat dissipation, and motivate further experimental works to study lattice thermal conductivity of TaN.
\end{abstract}

PACS numbers: 72.15.Jf, 71.20.-b, 71.70.Ej, 79.10.-n

Email:sandongyuwang@163.com Keywords: Lattice thermal conductivity; Group velocities; Phonon lifetimes

\section{INTRODUCTION}

Topological nontrivial phase, including topological insulator and semimetal, is one of the major advancements in condensed matter physics and material science ${ }^{1-15}$. The representative topological semimetals, such as Dirac semimetal $\left(\mathrm{Na}_{3} \mathrm{Bi}\right)^{6}$, Weyl semimetal $(\mathrm{TaAs})^{8,9,13,14}$ and nodal line semimetal $(\mathrm{ZrSiS})^{15}$, have been confirmed by angle-resolved photoemission spectroscopy (ARPES). In Dirac and Weyl semimetals, even-fold degenerate point can be observed in the momentum space ${ }^{6,8,11,12}$, such as four-fold degenerate Dirac point and two-fold degenerate Weyl point. Beyond Dirac and Weyl fermions, three, six- or eight-fold band crossings are proposed as new types of topological semimetals ${ }^{16}$. A band crossing between a doubly degenerate band and a nondegenerate band, namely three-fold degenerate crossing points, has been predicted in $\mathrm{TaN}, \mathrm{MoP}$ and $\mathrm{ZrTe}$ with WC-type structure ${ }^{17-19}$, and in in $\operatorname{InAs}_{0.5} \mathrm{Sb}_{0.5}{ }^{20}$. Experimentally, the MoP has been confirmed to be topological semimetal with triply degenerate nodal points (TDNPs), coexisting with the pairs of Weyl points ${ }^{2}$.

These topological semimetals may have substantial applications in electronics, spintronics and quantum computation $^{21}$. Efficient heat dissipation is a key factor for the reliable performance and stable function of electronic devices based on these topological semimetals, (a)
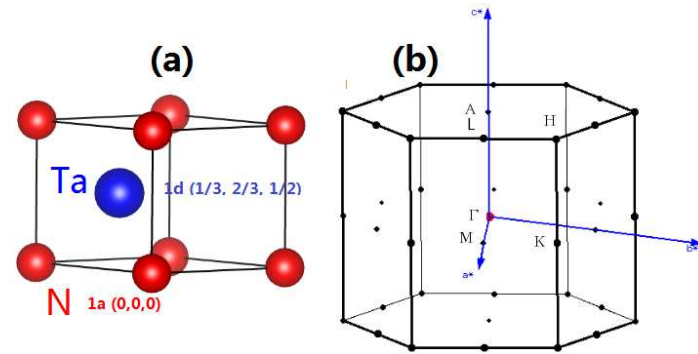

FIG. 1. (Color online)(a)The crystal structure of TaN in one unit cell; (b)the Brillouin zone with high-symmetry points.

especially for the high-power situations ${ }^{22}$. High lattice thermal conductivity is in favour of the high-performance thermal management ${ }^{23}$. The lattice thermal conductivity in TaAs, MoP and ZrTe has been calculated from a first principles calculation, showing obvious anisotropy along the a and c crystal axis ${ }^{24-27}$. However, their lattice thermal conductivities are relatively low, about $17 \sim 44$ $\mathrm{Wm}^{-1} \mathrm{~K}^{-1}$ at $300 \mathrm{~K}$, which is against efficient heat dissipation. Therefore, searching for topological semimetals with high lattice thermal conductivity is very necessary and interesting. In this work, the elastic properties of topological semimetal TaN are studied from firstprinciples calculations, and the phonon transport prop- 

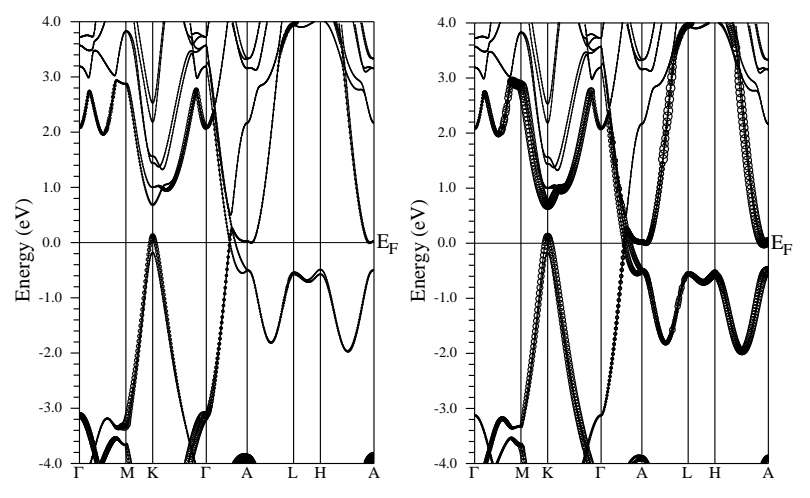

FIG. 2. The calculated energy band structures of TaN along high-symmetry paths using GGA+SOC, with fatted bands projected onto N (left) and Ta (Right) atoms .

erties are performed by solving the phonon Boltzmann transport equation. The calculated bulk modulus, shear modulus and $C_{44}$ suggest that $\mathrm{TaN}$ is a potential low compressible and hard material. The calculated lattice thermal conductivity is very higher than one of TaAs, $\mathrm{MoP}$ and $\mathrm{ZrTe}^{24-27}$, and the room-temperature lattice thermal conductivity is predicted to be $838.62 \mathrm{Wm}^{-1} \mathrm{~K}^{-1}$ and $1080.40 \mathrm{Wm}^{-1} \mathrm{~K}^{-1}$ along the a and $\mathrm{c}$ axis. This can be attributed to the large acoustic-optical frequency gap due to the large mass difference of Ta and N, producing inefficient scattering among acoustic and optical phonon modes. The mass difference factor suggests that $\mathrm{WC}$ and WN are potential topological materials with ultrahigh lattice thermal conductivity.

The rest of the paper is organized as follows. In the next section, we shall give our computational details about phonon transport. In the third section, we shall present elastic and phonon transport properties of TaN. Finally, we shall give our discussion and conclusions in the fourth section.

\section{COMPUTATIONAL DETAIL}

First-principles calculations are carried out within the projected augmented wave (PAW) method, as implemented in the VASP $\operatorname{code}^{28-30}$. The generalized gradient approximation of the Perdew-Burke-Ernzerhof (GGA$\mathrm{PBE})^{31}$ is adopted for the exchange-correlation functional with the plane-wave-cut-off energy of $500 \mathrm{eV}$, The $2 \mathrm{~s}$ and $2 \mathrm{p}$ electrons of $\mathrm{N}$, and $6 \mathrm{~s}$ and $5 \mathrm{~d}$ electrons of Ta are treated as valance ones. The energy convergences are less than $10^{-8} \mathrm{eV}$. The lattice thermal conductivity of TaN is calculated by solving linearized phonon Boltzmann equation with the single mode RTA, as implemented in the Phono3py code ${ }^{32}$. The lattice thermal conductivity can be expressed as

$$
\kappa_{L}=\frac{1}{N V_{0}} \sum_{\lambda} \kappa_{\lambda}=\frac{1}{N V_{0}} \sum_{\lambda} C_{\lambda} \nu_{\lambda} \otimes \nu_{\lambda} \tau_{\lambda}
$$

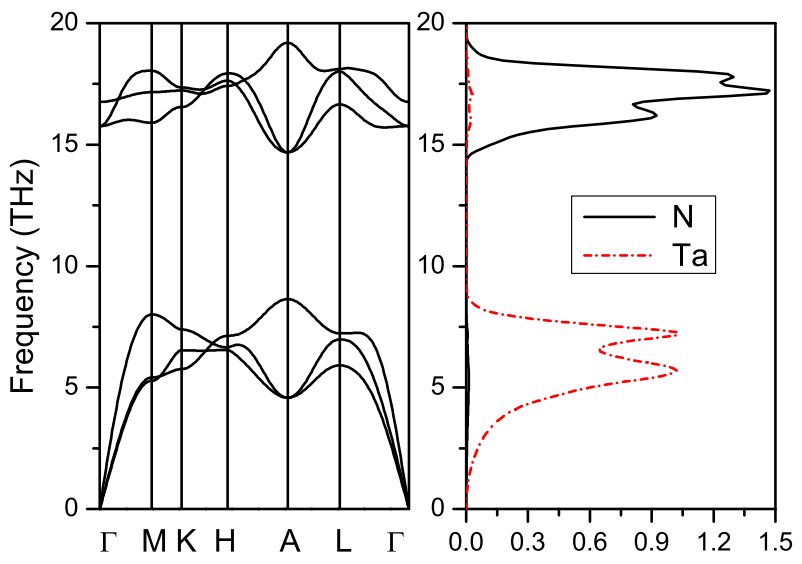

FIG. 3. (Color online)Phonon dispersion curve of TaN, along with atom partial density of states (PDOS).

where $\lambda$ is phonon mode, $N$ is the total number of $\mathrm{q}$ points sampling the Brillouin zone (BZ), $V_{0}$ is the volume of a unit cell, and $C_{\lambda}, \nu_{\lambda}, \tau_{\lambda}$ is the specific heat, phonon velocity, phonon lifetime. The phonon lifetime $\tau_{\lambda}$ can be attained by phonon linewidth $2 \Gamma_{\lambda}\left(\omega_{\lambda}\right)$ of the phonon mode $\lambda$ :

$$
\tau_{\lambda}=\frac{1}{2 \Gamma_{\lambda}\left(\omega_{\lambda}\right)}
$$

The $\Gamma_{\lambda}(\omega)$ takes the form analogous to the Fermi golden rule:

$$
\begin{gathered}
\Gamma_{\lambda}(\omega)=\frac{18 \pi}{\hbar^{2}} \sum_{\lambda^{\prime} \lambda^{\prime \prime}}\left|\Phi_{-\lambda \lambda^{\prime} \lambda^{\prime \prime}}\right|^{2}\left[( f _ { \lambda } ^ { \prime } + f _ { \lambda } ^ { \prime \prime } + 1 ) \delta \left(\omega-\omega_{\lambda}^{\prime}-\right.\right. \\
\left.\left.\omega_{\lambda}^{\prime \prime}\right)+\left(f_{\lambda}^{\prime}-f_{\lambda}^{\prime \prime}\right)\left[\delta\left(\omega+\omega_{\lambda}^{\prime}-\omega_{\lambda}^{\prime \prime}\right)-\delta\left(\omega-\omega_{\lambda}^{\prime}+\omega_{\lambda}^{\prime \prime}\right)\right]\right]
\end{gathered}
$$

in which $f_{\lambda}$ is the phonon equilibrium occupancy and $\Phi_{-\lambda \lambda^{\prime} \lambda^{\prime \prime}}$ is the strength of interaction among the three phonons $\lambda, \lambda^{\prime}$, and $\lambda^{\prime \prime}$ involved in the scattering.

The second- and third-order interatomic force constants (IFCs) are calculated by the supercell approach with finite atomic displacement of $0.03 \AA$. For secondorder harmonic IFCs, a $4 \times 4 \times 4$ supercell containing 128 atoms is used with k-point meshes of $2 \times 2 \times$ 2. Based on the harmonic IFCs, phonon dispersion of TaN can be calculated by Phonopy package ${ }^{33}$, which determines the allowed three-phonon scattering processes. The group velocity and specific heat can also be attained from phonon dispersion. For the third-order anharmonic IFCs, a $3 \times 3 \times 3$ supercells containing 54 atoms is used with k-point meshes of $3 \times 3 \times 3$. Based on third-order anharmonic IFCs, the three-phonon scattering rate can be attained, and further the phonon lifetimes can be calculated. To compute lattice thermal conductivities, the reciprocal spaces of the primitive cells are sampled using the $20 \times 20 \times 20$ meshes. 
TABLE I. The elastic constants $C_{i j}$, bulk $(B)$, shear $(G)$ and Young's ( $E_{x x}$ and $\left.E_{z z}\right)$ moduli (in GPa) of TaN, MoP and ZrTe.

\begin{tabular}{ccccccccccc}
\hline \hline Name & $C_{11}$ & $C_{12}$ & $C_{13}$ & $C_{33}$ & $C_{44}$ & $C_{66}$ & $B$ & $G$ & $E_{x x}$ & $E_{z z}$ \\
\hline \hline TaN & 566.40 & 128.23 & 62.41 & 706.39 & 215.02 & 219.09 & 260.26 & 233.16 & 534.04 & 695.17 \\
\hline MoP & 359.00 & 153.73 & 160.14 & 515.15 & 169.22 & 102.64 & 239.10 & 134.96 & 274.28 & 415.11 \\
\hline ZrTe & 140.82 & 58.78 & 88.81 & 201.11 & 110.36 & 41.02 & 102.50 & 61.26 & 97.83 & 122.07 \\
\hline \hline
\end{tabular}
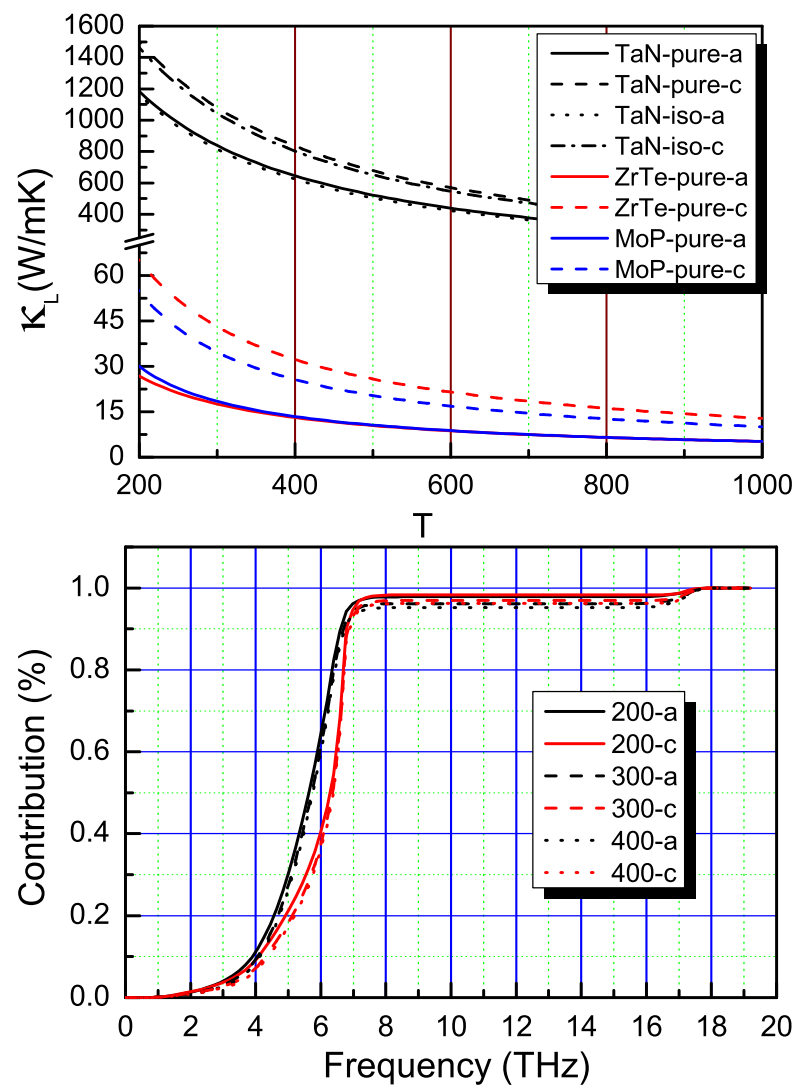

FIG. 4. (Color online) The lattice thermal conductivities of infinite (Pure and Isotope) TaN, ZrTe and MoP as a function of temperature, including a and c directions; The cumulative lattice thermal conductivity (200, 300 and $400 \mathrm{~K}$ ) of infinite (Pure) TaN divided by total lattice thermal conductivity with respect to phonon frequency, along a and c directions.

\section{MAIN CALCULATED RESULTS AND ANALYSIS}

The TaN shows WC-type crystal structure with space group $P \overline{6} m 2$ (No.187), and the crystal structure is shown in Figure 1. The MoP and $\mathrm{ZrTe}$ have the same crystal structure with $\mathrm{TaN}$. The Ta and $\mathrm{N}$ atoms occupy the 1d $(1 / 3,2 / 3,1 / 2)$ and $1 \mathrm{a}(0,0,0)$ Wyckoff positions, respectively. Experimentally, it can be synthesized at high pressure within a proper high temperature range. The experimental lattice constants $(a=b=2.9333 \AA, c=2.8844$ $\AA)^{34}$ are used to investigate elastic properties and lattice thermal conductivity of TaN. Firstly, the energy band structures of TaN with fatted bands projected onto $\mathrm{N}$ and Ta atoms are plotted in Figure 2. It can be seen that there are two TDNPs along $\Gamma$-A direction with Ta$\mathrm{d}$ character. Our calculated energy band structures agree well with previous report ${ }^{17}$. For MoP and $\mathrm{ZrTe}$, besides TDNPs, there are Weyl nodes in first BZ ${ }^{2,18}$.

Based on the experimental crystal structure, the elastic constants $C_{i j}$ of the TaN are calculated, which are listed in Table I, along with ones of $\mathrm{MoP}$ and $\mathrm{ZrTe}$. The elastic constants satisfy the following mechanical stability criteria ${ }^{35,36}$

$$
\begin{gathered}
C_{44}>0 \\
C_{11}>\left|C_{12}\right| \\
\left(C_{11}+2 C_{12}\right) C_{33}>2 C_{13}^{2}
\end{gathered}
$$

This indicates that the system of TaN is in a mechanical stable. Based on calculated elastic constants, the bulk, shear and Young's modulus can be obtained by Voigt-Reuss-Hill approximations. The Voigt's, Reuss's and Hill's bulk modulus can be calculated by the following equations:

$$
\begin{gathered}
B_{V}=\frac{1}{9}\left(2 C_{11}+C_{33}+2 C_{12}+4 C_{13}\right) \\
B_{R}=\left(2 S_{11}+S_{33}+2 S_{12}+4 S_{13}\right)^{-1} \\
B_{H}=\frac{1}{2}\left(B_{V}+B_{R}\right)
\end{gathered}
$$

The Voigt's, Reuss's and Hill's shear modulus can be attained by using these formulas:

$$
\begin{gathered}
G_{V}=\frac{1}{15}\left(2 C_{11}+C_{33}-C_{12}-2 C_{13}+6 C_{44}+3 C_{66}\right) \\
G_{R}=\left[\frac{1}{15}\left(8 S_{11}+4 S_{33}-4 S_{12}-8 S_{13}+6 S_{44}+3 S_{66}\right)\right]^{-1} \\
G_{H}=\frac{1}{2}\left(G_{V}+G_{R}\right)
\end{gathered}
$$

The Young's modulus $E_{i i}$ can be computed by the relationship:

$$
E_{i i}=1 / S_{i i}
$$



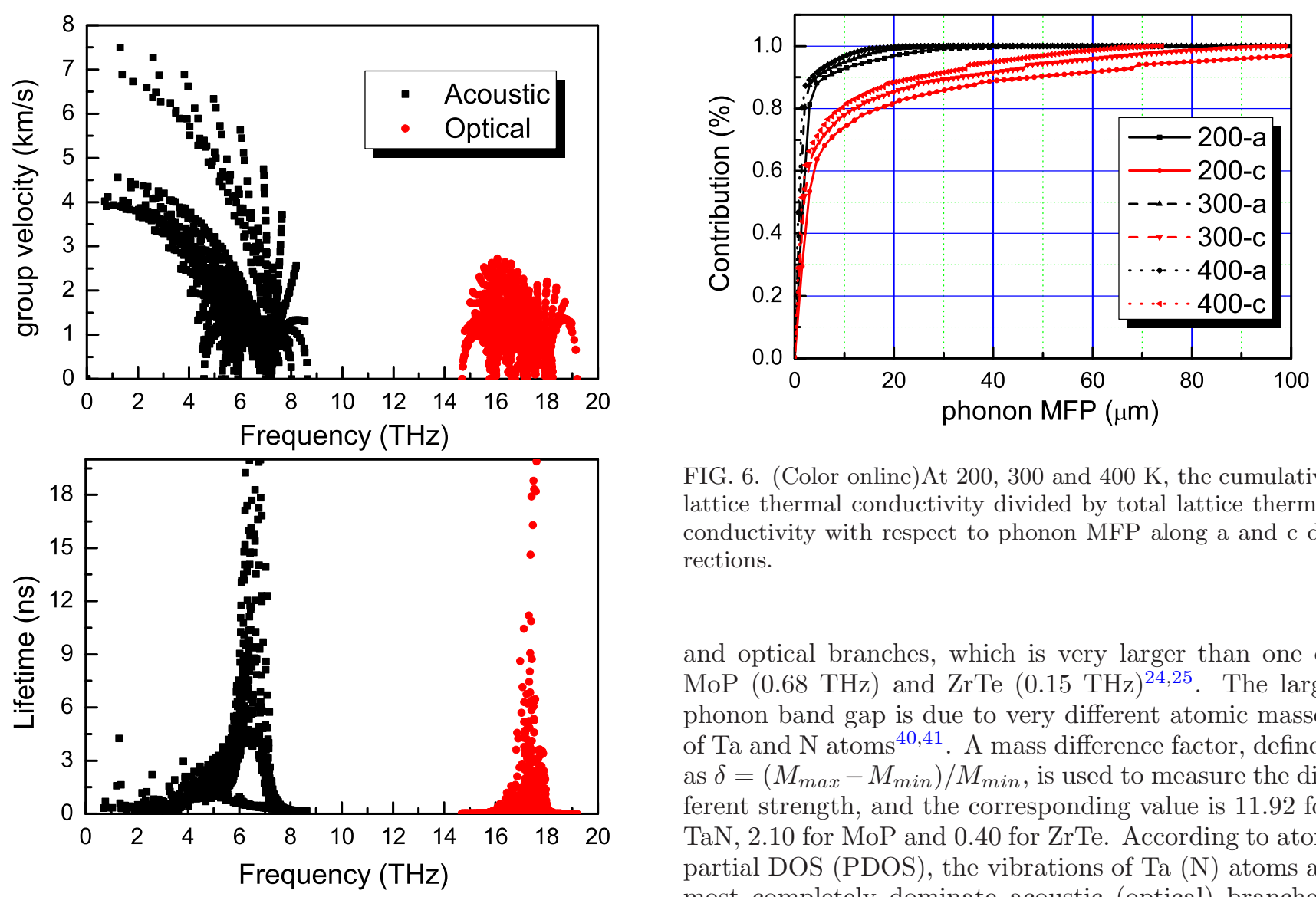

FIG. 6. (Color online)At 200, 300 and $400 \mathrm{~K}$, the cumulative lattice thermal conductivity divided by total lattice thermal conductivity with respect to phonon MFP along a and c directions.

and optical branches, which is very larger than one of $\mathrm{MoP}(0.68 \mathrm{THz})$ and $\mathrm{ZrTe}(0.15 \mathrm{THz})^{24,25}$. The large phonon band gap is due to very different atomic masses of Ta and $\mathrm{N}$ atoms ${ }^{40,41}$. A mass difference factor, defined as $\delta=\left(M_{\max }-M_{\min }\right) / M_{\min }$, is used to measure the different strength, and the corresponding value is 11.92 for TaN, 2.10 for MoP and 0.40 for $\mathrm{ZrTe}$. According to atom partial DOS (PDOS), the vibrations of Ta $(\mathrm{N})$ atoms almost completely dominate acoustic (optical) branches. These are familiar from the diatomic linear chain model,

FIG. 5. (Color online)The mode level phonon group velocities and phonon lifetimes (300K) of infinite (Pure) TaN in the first BZ.

The $S_{i j}$ are the elastic compliance constants. The related data are tabulated in Table I, together with ones of MoP and ZrTe. The $B / G$ can be used to measure material behaviour as ductile $(B / G>1.75)$ or brittle $(B / G<1.75)$. For TaN, the calculated $B / G$ ratio value is 1.12 , indicating that the brittle character is dominant. Bulk modulus or shear modulus can measure the hardness of materials ${ }^{37}$. The materials with higher bulk or shear modulus may be likely harder materials. The magnitude of the shear modulus $C_{44}$ may be a better hardness predictor for transition-metal carbonitrides ${ }^{38,39}$. Based on these criteria, TaN may be a incompressible and hard material. Experimental studies on structural and mechanical properties of TaN are strongly recommended.

6

Based on harmonic IFCs matrix, the phonon dispersion and and atom partial density of states (DOS) of TaN are plotted in Figure 3. No imaginary frequencies are observed in the phonon dispersion of TaN, indicating the thermodynamic stability of TaN. Two atoms per unit cell lead to 3 acoustic and 3 optical phonon branches. In contrast to MoP and ZrTe, a significant difference is observed. It is clearly seen that there is a phonon band gap of $6.05 \mathrm{THz}$ at the A point $(0,0, \pi / 2)$ between acoustic where acoustic (optical) branches are mainly contributed by the vibrations of by the larger (smaller) mass.

From harmonic and anharmonic IFCs, the intrinsic lattice thermal conductivity of TaN can be calculated by solving the linearized phonon Boltzmann equation within single-mode RTA method. Based on the formula proposed by Shin-ichiro Tamura ${ }^{42}$, the phonon-isotope scattering can be included. Along a and c directions, the lattice thermal conductivities of pure and isotopic $\mathrm{TaN}$ as a function of temperature are shown in Figure 4, together with ones of $\mathrm{ZrTe}$ and $\mathrm{MoP}$ for comparison. Similar to $\mathrm{ZrTe}$ and $\mathrm{MoP}$, the lattice thermal conductivity of TaN shows obvious anisotropy. It is clearly seen that the c-axis lattice thermal conductivity is very higher than a-axis one. The room-temperature lattice thermal conductivity of pure (isotopic) TaN along $\mathrm{a}$ and $\mathrm{c}$ axis is $838.62(814.96) \mathrm{Wm}^{-1} \mathrm{~K}^{-1}$ and 1080.40 (1044.06) $\mathrm{Wm}^{-1} \mathrm{~K}^{-1}$, respectively. The room temperature "isotope effect" is given by $P=\left(\kappa_{\text {pure }} / \kappa_{\text {iso }}-1\right)$, which is $2.90 \%$ along a axis, and $3.48 \%$ along $\mathrm{c}$ axis. These mean that phonon-isotope scattering has little effects on lattice thermal conductivity. Due to enhancement of phonon-phonon scattering, isotopic effect on lattice thermal conductivity gradually becomes weak with increasing temperature. An anisotropy factor ${ }^{26}$, defined as $\eta=\left(\kappa_{L}(c c)-\kappa_{L}(a a)\right) / \kappa_{L}(a a)$, can be used to measure the anisotropic strength. The $\eta$ for TaN is $28.83 \%$, which is smaller than that of MoP (88.5\%) and $\mathrm{ZrTe}(145.3 \%)$, 


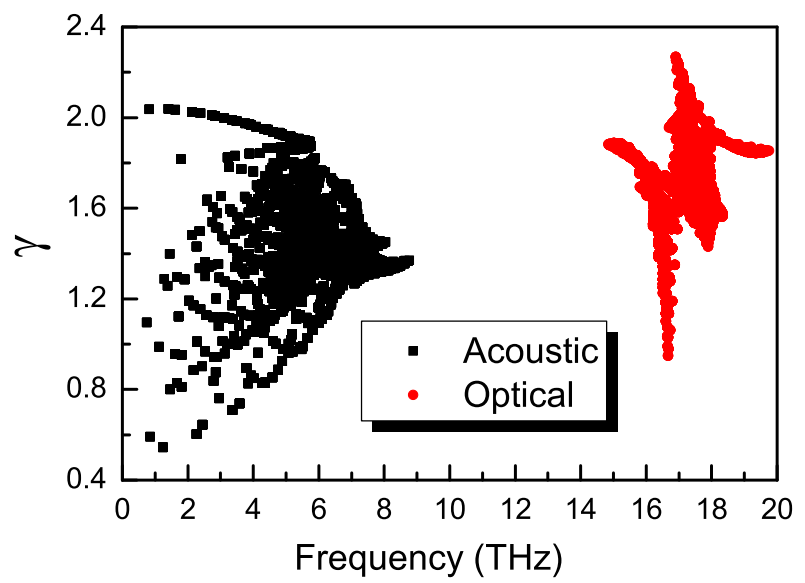

FIG. 7. (Color online)The mode level phonon Grüneisen parameters $(\gamma)$ of $\mathrm{TaN}$ in the first BZ.

implying weak anisotropy with respect to MoP and ZrTe. This can also be explained by the shear anisotropy ratio $A$, defined as $A=C_{44} / C_{66}{ }^{43}$. The calculated $A$ for TaN (0.98) is more closer to 1 than ones of MoP (1.65) and ZrTe (2.69). It is noted that the lattice thermal conductivity of TaN along a (c) direction is around 45 (31) times higher than that of MoP, and about 48 (25) times higher than one of ZrTe. The relation between lattice thermal conductivity and Young's modulus is $\kappa_{L} \sim \sqrt{E^{44}}$. It is found that the order of lattice thermal conductivity along a and c directions is consistent with one of Young's modulus. At 200, 300 and $400 \mathrm{~K}$, the cumulative lattice thermal conductivities divided by total lattice thermal conductivity with respect to frequency along a and c directions are shown in Figure 4. The cumulative thermal conductivity is defined by:

$$
\kappa^{c}(\omega)=\int_{0}^{\omega} \Sigma_{\lambda} \kappa_{\lambda} \delta\left(\omega_{\lambda}-\omega^{\prime}\right) d \omega^{\prime}
$$

It is clearly seen that nearly all lattice thermal conductivity is made up of the acoustic phonon branches for three considered temperature. It is noted that the cumulative lattice thermal conductivities divided by total lattice thermal conductivity with respect to frequency has weak temperature dependence. The slope along a direction is larger than that along c direction, which means that low frequency phonon has larger contribution to total lattice thermal conductivity for a than c direction.

To gain more insight into high lattice thermal conductivity of TaN, we show the mode level phonon group velocities and lifetimes in Figure 5. In long-wavelength limit, the largest phonon group velocity of TA1, TA2 and LA branches is $3.91 \mathrm{kms}^{-1}, 4.12 \mathrm{kms}^{-1}$ and $6.89 \mathrm{kms}^{-1}$, respectively. These are larger than ones of $\mathrm{ZrTe}^{25}$, but smaller than ones of $\mathrm{MoP}^{24}$. It is clearly seen that the most of group velocities of acoustic branches are higher than those of optical branches. It is found that most of phonon lifetimes of TaN are very longer than ones of MoP and $\mathrm{ZrTe}^{24,25}$, which leads to very higher lattice thermal conductivity for TaN than MoP and ZrTe. Unexpectedly, the phonon lifetimes near 6 and $17 \mathrm{THz}$ become very large. This can be understood by very large frequency gap between the optical and acoustic phonon branches. The frequency gap $(6.05 \mathrm{THz})$ is close to the range of acoustic phonons $(8.64 \mathrm{THz})$. While heat is transmitted primarily by the acoustic branches, the optical branches provide important scattering channels for the acoustic modes, particularly through acoustic + acoustic $\rightarrow$ optical scattering. Because of the requirement on energy conservation for phonon-phonon scattering, the annihilation process of two acoustic phonon modes into one optical one becomes ineffective (such annihilation process is not totally prohibited.) caused by acoustic-optical gap. As a result, the weaker phonon-phonon scattering rate is produced, and then results in long phonon lifetimes, giving rise to a much high lattice thermal conductivity. The very high thermal conductivity is found in $\mathrm{BAs}^{45}$ and $\mathrm{AlSb}^{46}$, which is also due to a large frequency gap.

The size dependence of lattice thermal conductivity of $\mathrm{TaN}$ can be reflected by the cumulative lattice thermal conductivity with respect to MFP, which shows how phonons with different MFP contribute to the thermal conductivity. The MFP cumulative lattice thermal conductivity is defined as:

$$
\begin{gathered}
\kappa^{c}(l)=\int_{0}^{l} \Sigma_{\lambda} \kappa_{\lambda} \delta\left(l_{\lambda}-l^{\prime}\right) d l^{\prime} \\
l_{\lambda}=\left|l_{\lambda}\right|=\left|\nu_{\lambda} \otimes \tau_{\lambda}\right|
\end{gathered}
$$

At 200, 300 and $400 \mathrm{~K}$, the cumulative lattice thermal conductivity divided by total lattice thermal conductivity with respect to MFP along a and c directions are shown in Figure 6. It is clearly seen that the cumulative lattice thermal conductivity of TaN along both a and c axis approaches saturation value with MFP increasing. With the increasing temperature, the critical MFP gradually decreases. At $300 \mathrm{~K}$, phonons with MFP larger than 20 (84) $\mu \mathrm{m}$ along a (c) direction has little contribution to the total lattice thermal conductivity. At room temperature, phonons with MFP smaller than 1 (2) $\mu \mathrm{m}$ along $\mathrm{a}(\mathrm{c})$ direction contribute around half to the total lattice thermal conductivity. These results mean that the lattice thermal conductivity along c direction is tuned more easily than that along a direction.

\section{DISCUSSIONS AND CONCLUSION}

The MoP, ZrTe and TaN have the same crystal structure, and their lattice thermal conductivities show obvious anisotropy along a and c directions, where the lattice thermal conductivity along $\mathrm{c}$ direction is larger than that along a direction. However, a very higher lattice thermal conductivity of TaN is observed than that of MoP and ZrTe. Traditionally, a low Debye temperature $T_{D}$ indicates low lattice thermal conductivity. All phonon 
TABLE II. For TaN, MoP and ZrTe, the longitudinal, transversal and average sound speed $\left(v_{L}, v_{T}\right.$ and $v_{A}$ in $\left.\mathrm{km} / \mathrm{s}\right)$, Debye temperature $\left(T_{D}\right.$ in $\left.\mathrm{K}\right)$; the frequency gap between acoustic and optical phonons (Gapao in THz); the a-axis, c-axis and average lattice thermal conductivity $\left(\kappa_{a a}, \kappa_{c c}\right.$ and $\kappa_{A}$ in $\left.\mathrm{Wm}^{-1} \mathrm{~K}^{-1}\right)$.

\begin{tabular}{lcccccccc}
\hline \hline Name & $v_{L}$ & $v_{T}$ & $v_{A}$ & $T_{D}$ & Gapao & $\kappa_{a a}$ & $\kappa_{c c}$ & $\kappa_{A}$ \\
\hline \hline TaN & 6.11 & 3.79 & 4.80 & 647.92 & 6.05 & 838.62 & 1080.4 & 919.21 \\
\hline MoP & 7.48 & 4.36 & 5.63 & 687.17 & 0.68 & 18.41 & 34.71 & 23.84 \\
\hline ZrTe & 4.92 & 3.12 & 3.92 & 405.78 & 0.15 & 17.56 & 43.08 & 26.07 \\
\hline \hline
\end{tabular}

modes are excited with the temperature above $T_{D}$, which can give rise to strong three-phonon scattering, and then suppress lattice thermal conductivity. When the temperature is below $T_{D}$, some phonon modes begin to be frozen out $^{47}$. The Debye temperature can be obtained from the average sound velocity using the following equation ${ }^{48}$ :

$$
T_{D}=\left(\frac{3 N}{4 \pi V_{0}}\right)^{1 / 3} \frac{h v_{A}}{k_{B}}
$$

where $\mathrm{N}$ denotes the number of atom in the primitive unit cell, $V_{0}$ denotes the unit cell volume, $h$ and $k_{B}$ denote the Planck's and Boltzmann's constants. The average sound speed $v_{A}$ can be calculated from the longitudinal and transversal sound velocities, $v_{L}$ and $v_{T}$.

$$
v_{A}=\left[\frac{1}{3}\left(\frac{1}{v_{L}^{3}}+\frac{2}{v_{T}^{3}}\right)\right]^{-1 / 3}
$$

The longitudinal and transversal sound velocities, $v_{L}$ and $v_{T}$, are related to the bulk, shear modulus and the density of the material, $B, G$ and $\rho$.

$$
\begin{gathered}
v_{L}=\sqrt{(B+4 G / 3) / \rho} \\
v_{T}=\sqrt{G / \rho}
\end{gathered}
$$

The longitudinal, transversal, average sound speed and Debye temperature of TaN, MoP and ZrTe are listed in Table II. Assumed from Debye temperature, the lattice thermal conductivity of TaN should be lower than one of MoP, and should be slightly higher than that of ZrTe. In contrast to this straightforward prediction, the calculated lattice thermal conductivity of TaN is dozens of times higher than one of MoP or ZrTe. Based on the formula proposed by Slack ${ }^{49}$, four factors, including the average atomic mass, interatomic bonding, crystal structure and anharmonicity, determine the lattice thermal conductivity. A high Debye temperature can be produced by low average atomic mass and strong interatomic bonding, leading to a high thermal conductivity. Mode Grüneisen parameters $\gamma$ can measure the strength of anharmonic interactions, determining the intrinsic phononphonon scattering. The larger $\gamma$ gives rise to stronger anharmonic phonon scattering, leading to lower lattice thermal conductivity. The mode level Grüneisen parameters of TaN are plotted in Figure 7. The average Grüneisen
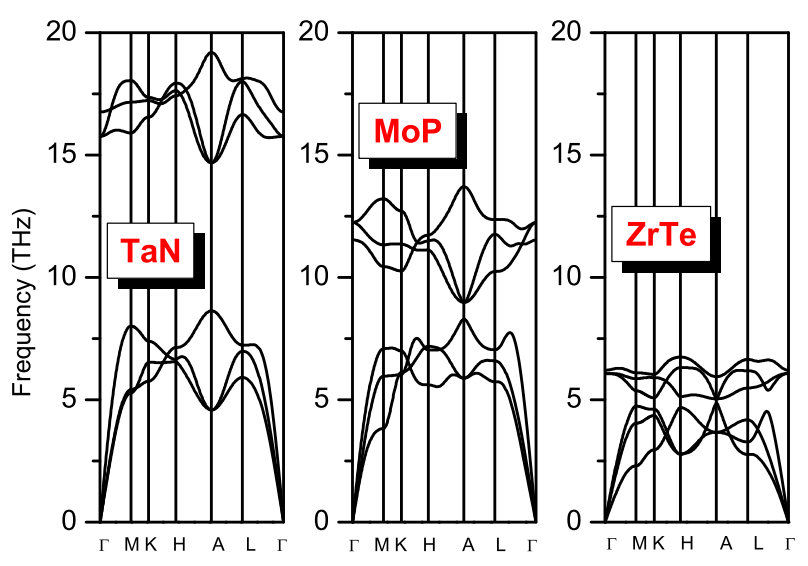

FIG. 8. (Color online)Phonon dispersion curves of TaN, MoP and $\mathrm{ZrTe}$.

parameter is 1.60 , which is close to 1.57 of $\mathrm{MoP}^{24}$ and 1.52 of $\mathrm{ZrTe}^{25}$. Therefore, other factors should be found to explain very high lattice thermal conductivity in TaN. Although TaN, MoP and ZrTe have similar outlines of phonon dispersion (see Figure 8), a significant difference is found. A very large frequency gap $(6.05 \mathrm{THz})$ between the optical and acoustic phonon branches in TaN is observed, while the gap is only $0.68 \mathrm{THz}$ for $\mathrm{MoP}$ and 0.15 $\mathrm{THz}$ for $\mathrm{ZrTe}$. The large thermal conductivity of TaN is due to the large acoustic-optical frequency gap caused by the large mass difference of Ta and N, which can lead to inefficient scattering among acoustic and optical phonon modes. The frequency gap between acoustic and optical phonons, a-axis, c-axis and average lattice thermal conductivities $\left(\kappa_{A}=\left(\kappa_{a a}+\kappa_{b b}+\kappa_{c c} / 3\right)\right.$ of $\mathrm{TaN}, \mathrm{MoP}$ and ZrTe are listed in Table II. Therefore, the extremely large frequency gap has a dramatic effect on lattice thermal conductivity of materials. Recently, many materials with WC-type crystal structure have been predicted as topological metal candidates ${ }^{6}$. The mass difference factor $\delta$ for these topological metals are plotted in Figure 9. The large $\delta$ can lead to large acoustic-optical gap, inducing large lattice thermal conductivity by restricting acoustic+acoustic $\rightarrow$ optical scattering. It is clearly seen that $\delta$ of WC and WN is larger than 10, being close to one of TaN. So, WC and WN may be potential candidates with ultrahigh lattice thermal conductivity.

In summary, the elastic and phonon transport properties of TaN are investigated by combining the first- 


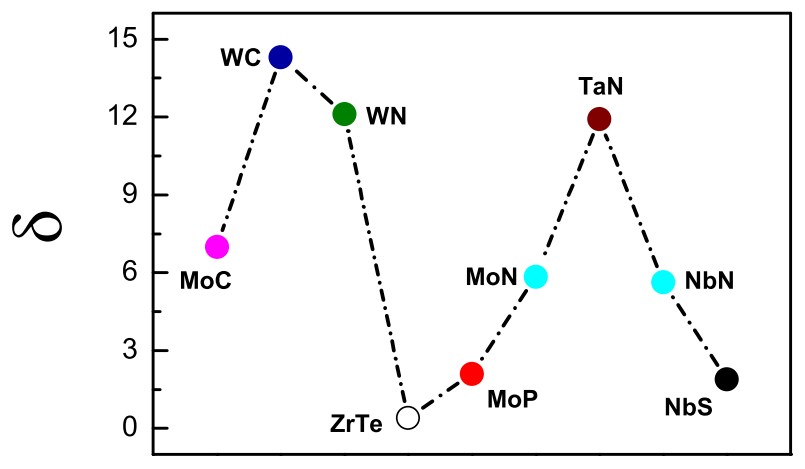

FIG. 9. (Color online)The mass difference factor of a list of topological metals with WC-type crystal structure.

principles calculations and semiclassical Boltzmann transport theory. Based on elastic tensor components $C_{i j}$, the mechanical stability of TaN is confirmed by mechanical stability criteria. The bulk modulus, shear modulus, Young's modulus, the longitudinal sound speed, transversal sound speed and Debye temperature are also attained, according to calculated $C_{i j}$. It is predicted that TaN may be a potential low compressible and hard ma- terial, based on calculated bulk modulus, shear modulus, and $C_{44}$. It is found that TaN has ultrahigh lattice thermal conductivity, showing an obvious anisotropy along the a and c crystal axis. The extremely large frequency gap in TaN strongly restricts acoustic+acoustic $\rightarrow$ optical scattering through the energy conservation, which leads to ultrahigh lattice thermal conductivity in TaN. Calculated results show that isotope scattering has little effect on the lattice thermal conductivity of TaN, and phonons with MFP larger than 20 (80) $\mu \mathrm{m}$ along a (c) direction have little contribution to the total lattice thermal conductivity. Our works shed light on elastic and phonon transport properties of TaN, and help to seek ultrahigh lattice thermal conductivity in topological semimetals by large frequency gap, such as WC and WN.

\section{ACKNOWLEDGMENTS}

This work is supported by the National Natural Science Foundation of China (Grant No.11404391). We are grateful to the Advanced Analysis and Computation Center of CUMT for the award of CPU hours to accomplish this work.
1 R. M. Lutchyn, J. D. Sau and S. D. Sarma, Phys. Rev. Lett. 105, 077001 (2010).

2 B. Q. Lv, Z. L. Feng, Q. N. Xu et al., Nature 546, 627 (2017).

3 C. Fang, H. M. Weng, X. Dai and Z. Fang, Chinese Phys. B 25, 117106 (2016).

${ }^{4}$ H. J. Zhang, C. X. Liu, X. L. Qi, X. Dai, Z. Fang and S. C. Zhang, Nat. Phys. 5, 438 (2009).

${ }^{5}$ M. Z. Hasan and C. L. Kane, Rev. Mod. Phys. 82, 3045 (2010).

6 B. Q. Lv, H. M. Weng, B. B. Fu, X. P. Wang, H. Miao, J. Ma, P. Richard, X. C. Huang, L. X. Zhao, G. F. Chen, Z. Fang, X. Dai, T. Qian, and H. Ding, Phys. Rev. X 5, 031013 (2015).

7 X. L. Qi and S. C. Zhang, Rev. Mod. Phys. 83, 1057 (2011).

${ }^{8}$ L. X. Yang, Z. K. Liu and Y. Sun et al., Nat. Phys. 11, 728 (2015).

${ }^{9}$ S. Y. Xu et al. Nature Phys. 11, 748 (2015).

10 S. Y. Xu et al. Science Advances 10, e1501092 (2015).

11 Z. J. Wang, Y. Sun, X. Q. Chen, C. Franchini, G. Xu, H. M. Weng, X. Dai and Z. Fang, Phys. Rev. B 85, 195320 (2012)

12 Z. K. Liu, B. Zhou and Y. Zhang et al., Science 343, 864 (2014).

13 S. M. Huang et al. Nat. Commun. 6, 7373 (2015).

14 S. Y. Xu et al. Science 349, 613 (2015).

15 L. M. Schoop, M. N. Ali, C. Stra $\beta$ r, A. Topp, A. Varykhalov, D. Marchenko, V. Duppel, S. S. P. Parkin, B. V. Lotsch, and C. R. Ast, Nat. Commun. 7, 11696 (2016).

16 B. Bradlyn, J. Cano, Z. Wang, M. G. Vergniory, C. Felser, R. J. Cava and B. A. Bernevig, Science 353, aaf5037 (2016).

17 H. Weng, C. Fang, Z. Fang and X. Dai, Phys. Rev. B 93,
241202(R) (2016).

18 H. Weng, C. Fang, Z. Fang and X. Dai, Phys. Rev. B 94, 165201 (2016).

19 Z. M. Zhu, G. W. Winkler, Q. S. Wu, J. Li and A. A. Soluyanov, Phys. Rev. X 6, 031003 (2016).

20 G. W. Winkler, Q. S. Wu, M. Troyer, P. Krogstrup and A. A. Soluyanov, PRL 117, 076403 (2016).

21 Z. Li, H. Chen, S. Jin, D. Gan, W. Wang, L. Guo, X. Chen, Cryst. Growth Des. 16, 1172 (2016).

22 N. Han, T. V. Cuong, M. Han, B. D. Ryu, S. Chandramohan, J. B. Park, J. H. Kang, Y. J. Park, K. B. Ko, H. Y. Kim, H. K. Kim, J. H. Ryu, Y. S. Katharria, C. J. Choi, and C. H. Hong, Nat. Commun. 4, 1452 (2013).

23 G. Z. Qin, Z. Z. Qin, H. M. Wang and M. Hu, Phys. Rev. B 95, 195416 (2017).

24 S. D. Guo, J. Phys.: Condens. Matter 29435704 (2017).

25 S. D. Guo, Y. H. Wang and W. L. Lu, New J. Phys. in press (2017)

26 T. Ouyang, H. P. Xiao, C. Tang, M. Hu and J. X. Zhong, Phys. Chem. Chem. Phys. 18, 16709 (2016).

27 B. Peng, H. Zhang, H. Z. Shao, H. L. Lu, D. W. Zhang, H. Y. Zhua, Nano Energy 30, 225 (2016).

28 G. Kresse, J. Non-Cryst. Solids 193, 222 (1995).

29 G. Kresse and J. Furthmüller, Comput. Mater. Sci. 6, 15 (1996).

30 G. Kresse and D. Joubert, Phys. Rev. B 59, 1758 (1999).

31 J. P. Perdew, K. Burke and M. Ernzerhof, Phys. Rev. Lett. 77, 3865 (1996).

32 A. Togo, L. Chaput and I. Tanaka, Phys. Rev. B 91, 094306 (2015).

33 A. Togo, F. Oba, and I. Tanaka, Phys. Rev. B 78, 134106 (2008).

34 A. Friedrich, W. Morgenroth, L. Bayarjargal, E. A. Juarez- 
Arellano, B. Winkler, and Z. Konpkov, High Press. Res. 33, 633 (2013).

35 H. M. Ledbetter, J. Phys. Chem. Ref. Data 6, 1181 (1977).

36 Z. J. Wu, E. J. Zhao, H. P. Xiang, X. F. Hao, X. J. Liu and J. Meng, Phys. Rev. B 76, 054115 (2007).

37 D. M. Teter, MRS Bull. 23, 22 (1998).

38 S. H. Jhi, J. Ihm, S. G. Louie, and M. L. Cohen, Nature 399, 132 (1999).

39 Z. G. Wu, X. J. Chen, V. V. Struzhkin and R. E. Cohen, Phys. Rev. B 71, 214103 (2005).

40 L. Lindsay, D. A. Broido and T. L. Reinecke, Phys. Rev. Lett. 111, 025901 (2013).

41 X. Gu and R. Yang, Appl. Phys. Lett. 105, 131903 (2014).
42 S.I. Tamura, Phys. Rev. B, 27, 858 (1983).

43 J. Y. Wang and Y. C. Zhou, Phys. Rev. B 69, 144108 (2004).

44 W. Kim, J. Mater. Chem. C 3, 10336 (2015).

45 L. Lindsay, D. Broido, and T. Reinecke, Phys. Rev. Lett. 111, 025901 (2013).

${ }^{46}$ L. Lindsay, D. Broido, and T. Reinecke, Phys. Rev. B 87, 165201 (2013).

47 T. Nakashima and Y. Umakoshi, Philos. Mag. Lett. 66, 317 (1992).

48 P. Ravindran, L. Fast, P. A. Korzhavyi, B. Johnnsson, J.Wills, and O. Eriksson, J. Appl. Phys. 84, 4891 (1998).

49 G. A. Slack, J. Phys. Chem. Solids 34, 321 (1973). 